\title{
Oculomotor-related measures but not distance visual acuity are predictive of reading ability in first graders
}

\author{
Avi Portnoy ${ }^{1}$ and Sharon Gilaie-Dotan ${ }^{1,2, *}$ \\ ${ }^{1}$ School of Optometry and Vision Science, Faculty of Life Science, Bar Ilan University, Ramat Gan, \\ Israel \\ ${ }^{2}$ Institute of Cognitive Neuroscience, UCL, London, UK
}

Keywords: visual acuity, VA, OMD, oculomotor movements disorders, reading, King-Devick,

DEM, NSUCO, optometric examinations, vision screening

* Correspondence: Sharon Gilaie-Dotan, School of Optometry and Vision Science, Bar Ilan

University, Ramat Gan, 5290002, Israel, shagido@gmail.com 


\section{Abstract}

Vision screening in junior schools around the globe are often limited to distance visual acuity $(\mathrm{dVA})$. Oculomotor dysfunction (OMD) is an umbrella term that includes abnormalities in comfortable and accurate control of the oculomotor system (fixation, pursuits and saccades) and can exist despite normal dVA. Since we assumed that a basic prerequisite for successful reading is that the ocular sensory-motor functions perform in flawless harmony with effortless automaticity, we hypothesized that OMD but not dVA would have profound effect on comfortable and efficient acquisition of effective reading skills. Consequently, we retrospectively compared independently obtained (double blind) reading evaluations and extensive optometric screening examinations of a class of 28 first graders. We found, as we hypothesized, that performance on optometric oculomotor tests, but not dVA, were predictive of reading test performance (both speed and accuracy) according to the standardized national reading norms. Our results suggest reevaluating the effectiveness of dVA as a marker of visual readiness for scholastic achievements. We propose including OMD related assessments as part of a paradigm shift in both vision screening in early readers as well as potential treatment protocols for those pupils struggling to acquire efficient reading skills. 


\section{Introduction}

According to the Annie E Casey Foundation, in 2017, only 35\% of children starting fourth grade in the US had reading skills that were proficient or above proficient level relative to their age ${ }^{1}$. Furthermore, sociologist Donald Hernandez found that the risk of leaving school without a diploma increases by $400 \%$ if a child enters fourth grade without proficient reading skills ${ }^{2}$. Acquiring reading skills would therefore seem key to young adults finishing high school.

Reading is a complex process that depends on an effective ocular sensory-motor platform whilst simultaneously relying on high level visual-perceptual, cognitive, language and comprehension skills. During reading the visual system relies on high-level binocular control to ensure that the two eyes remain converged on the plane of the printed page whilst simultaneously shifting from word to word causing a relative adjustment in angle of each eye. Similarly, the letters must be kept in focus, remaining sensitive to changes in demand as the eye shifts to lines closer or further from the reader on the $Z$ axis. This must be done for prolonged periods of time despite using a muscle structure (smooth muscle) designed for rapid brief actions. All of these are tremendously intricate and highly demanding tasks that we expect to take place with effortless automaticity. With regard to oculomotor control, prior to performing a saccade the visual system calculates the location in space to which the reader's eyes are to be relocated. This is achieved with precision in a seemingly effortless task for much of the population who enjoy effective oculomotor function.

Oculomotor dysfunction (OMD) is an umbrella term that includes abnormalities in comfortable and accurate control of the oculomotor system, including fixation, saccades and pursuits. The 
prevalence of OMD in school aged 'good' readers is estimated to be $22-24 \%^{3,4}$ compared to as high as $95 \%$ in child populations diagnosed with a reading difficulty ${ }^{3}$. For those suffering from OMD saccades can overshoot, causing the eyes to land further than the designated target, or undershoot, causing the eyes to land short of the designated target. Both overshooting and undershooting are typically followed by smaller corrective saccades. OMD might also include unintentional motor responses to ocular movement (aka oculomotor overflow) of the jaw, tongue, head or body that accompany pursuits or saccadic movements. The extreme demands of reading on the oculomotor system may incapacitate a new reader who suffers from OMD, impacting on both reading speed and accuracy. It is assumed that the root cause of many OMD cases is the lack of effective integration of sensory input, higher level decision making, and appropriate oculomotor response rather than being a purely neuromotor or sensory level deficit.

It is clear that visual function deficits can adversely influence reading acquisition. Research shows that untreated far-sightedness (hypermetropia) ${ }^{5}$, poor accommodative control ${ }^{6,7}$ as well as poor functional binocular control ${ }^{7,8}$, severely impact reading ability and comfort ${ }^{9,10}$.

There is a plethora of evidence in the literature supporting a connection between ocular control and its impact on reading ability ${ }^{11-15}$.Despite this evidence, the prevalent testing protocols in vision amongst first graders in multiple European countries ${ }^{16}$ includes distance visual acuity (dVA) but typically omits screening for fixation, saccades, accommodation and convergence skills.

We hypothesized that a basic prerequisite for successful reading is that the ocular sensorymotor functions involved should work in flawless harmony, and therefore, OMD, nut not dVA, would have a profound effect on reading acquisition. 
Consequently, we retrospectively compared the results of a comprehensive optometric examination of a class of 28 first graders to their independently obtained reading evaluations. This optometric examination was performed by an optometrist who was blind to the children's school performance, and reading was assessed by a teacher who was blind to the optometric screening results. The optometric evaluation included dVA and several standard developmental optometric tests related to OMD, including King-Devick ${ }^{17}, \mathrm{DEM}^{18,19}$, and the Northeastern State University College of Optometry (NSUCO) ${ }^{20}$ oculomotor test. The reading assessment included reading speed and accuracy according to national standardized tests ${ }^{21}$. Specifically, we focused on examining the relationship between dVA or OMD-related measures and reading performance.

\section{Methods}

\section{Participants}

The cohort consisted of twenty-eight first grade children (17 boys and 11 girls) aged six and seven years in an Israeli school. The parents of the children, all in the same class, were offered the opportunity for their children to undergo an optometric screening examination in school and were asked to provide their consent. Out of a class of thirty children, all but 2 parents provided consent that their child participate in the optometric examination. The visual function screening assessment was performed by a certified optometrist and took roughly forty minutes per child. In addition, all the children underwent a comprehensive reading examination including reading speed and accuracy, performed by a reading teacher a couple of weeks after the optometric evaluation. Importantly, the teacher was not informed of the optometric results 
until a few weeks after the whole class's reading assessments were completed, and the optometrist was not informed of the reading assessment results until after the optometric examinations were completed. The Bar Ilan University ethics committee approved a retrospective analysis of the data. The children wore their current daily refractive correction for each of the optometric tests below. If visual acuity was found to be $6 / 12$ or worse in either eye with their current spectacle correction $(n=4)$, the child was referred to an optometrist for further refractive testing or an ophthalmologist for a full ocular health exam (based on retinoscopy results). In such cases $(n=4)$, the child's visual skills were then assessed several weeks later with any newly prescribed spectacle prescription.

\section{Visual screening examination}

The visual screening examination was performed by a certified optometrist that has practiced optometry for over 8 years. Each child's optometric screening assessment took approximately 40 minutes and included the following standardized optometric tests carried out in this order: Visual acuity (VA) near and distance, over refraction - retinoscopy, cover testing (distance and near), negative fusional vergence (NFV) at near, positive fusional vergence (PFV) at near, near point of convergence (NPC), stereopsis, accommodative amplitude (right eye (RE) only), accommodative facility (RE only), NSUCO, Developmental Eye Movement test (DEM) and KingDevick test. Lighting was provided by overhead fluorescent fixtures as well as sun light from windows. For the dVA testing and retinoscopy, the fluorescent lighting was extinguished, and the sunlight was partially blocked by curtains leaving the room dimly lit ${ }^{22}$.

Distance visual acuity (VA) was performed using a standard acuity chart projected onto a wall 6 meters from the child and performed both monocularly and binocularly ${ }^{22}$.

Near VA was assessed at $40 \mathrm{~cm}$ monocularly and binocularly using a near VA test card. 
Retinoscopy is an objective assessment of the child's refractive status ${ }^{23}$. This was used to ensure the child's current habitual spectacle prescription was not under or over corrected by more than 0.5 diopters (D) of myopia (short sightedness), 1.5D hyperopia (far sightedness) or 0.75D astigmatism $^{24}$. If it was, they were referred to get corrective spectacles before continuing $(n=4)$.

DEM test was used to assess saccadic accuracy and speed as well as assessing for rapid automatic naming (RAN) challenges ${ }^{25}$. It was performed according to the guidelines provided with the test ${ }^{26}$.

The King-Devick test, another test used to assess saccadic speed and accuracy and was performed according to the testing protocol that is attached to the screening tool ${ }^{27}$.

NSUCO (Northeastern State University College of Optometry) oculomotor test was carried out according to the manual supplied by the OEP foundation ${ }^{28}$. We chose to assess whether the head movement subtest during saccades were predictive of reading accuracy. The inability to dissociate ocular movement from head movement is a typical sign of OMD. Head movements demand more energy than ocular movements and are not precise enough to meet the demands necessary for accurate reading. Lastly there is no saccadic suppression that takes place during head movements. This often results in people with OMD perceiving letters as moving on the page.

\section{Reading assessment examination}

Reading of each child was tested by an experienced reading teacher. The teacher used the agebased norm-referenced tests provided by the Israeli Ministry of Education and followed their fixed protocol for testing ${ }^{21}$. The assessment comprised 10 different subtests assessing reading and writing skills including accuracy, speed, phonological awareness, and reading comprehension. 
In our retrospective analysis we chose to focus on subtest 8 which assesses reading speed and accuracy since it best simulates visual demands of daily classroom reading. The test demands reading text aloud while the teacher evaluates both accuracy and speed. This subtest does not examine comprehension. The text is 77 words long presented in 8 widely spaced lines typically taking at that age 60 to 266 seconds to complete. The teacher marks all the words correctly read by the child including self-correction or inaccuracies which match everyday speech. According to the national standards, a score of 69-77 words read correctly is considered a pass, a score of 5868 words correct demands follow up and a score below 57 words correct is a fail. The teacher then assessed the severity and type of reading errors including morphological, morph-syntactic, word switching etc. With regards to reading speed the teacher measures the amount of time it takes for the child to read the 77 words. A score of less than 195 seconds is considered a pass, a score between 196 and 265 seconds demands follow up and a score of over 266 seconds is deemed a fail.

\section{Analyses}

To assess the relationship between visual optometric measures and reading performance (speed/accuracy), we first correlated oculomotor measures with reading measures (saccadic speed with reading speed and saccadic accuracy with reading accuracy). Furthermore, the class was divided into two halves according to specific optometric performances (visual acuity and then oculomotor functions) and in each such analysis, for each half (lower and higher in vision scores) we examined their achievements on the reading test (the proportion of children who passed, needed follow up, or failed). 


\section{Results}

\section{Distance VA}

Whilst we hypothesized that dVA would not be correlated with reading speed or accuracy, it is currently the only formal first grade optometric check enforced by the Ministry of Health in Israel, ${ }^{29}$ as is the case in many other countries ${ }^{16}$. To assess whether dVA was predictive of reading performance, we examined whether children with superior dVA would perform better on the reading tests than children with poorer dVA. To that end we first correlated the reading speed and accuracy performance (subtest no. $8^{21}$ ) with dVAs and found that dVA was not significantly predictive of reading accuracy $(R=-0.082, t(26)=-0.42, p=0.68)$ or of reading speed $(R=0.25, t(26)=1.32, p=0.20)$. Figure $1 A$ presents reading performance across 3 reading subtests (reading a section from a short story aloud (subtest no. 8), reading a column of unfamiliar words (subtest no. 7), or a column of familiar words (subtest no. 6) sorted by dVA. As can be seen, higher dVA scores do not seem to be indicative of superior reading performance. We also compared the proportions of "pass", "requires follow up" and "fail" scores for reading accuracy and speed according to national reading standards ${ }^{21}$ (subtest 8 ) between children with normal dVA $(=6 / 6)$ and children with lower dVA $(<6 / 6)$. We focused on subtest 8 since it best simulates visual demands of daily classroom reading. In these analyses we found that amongst children with normal dVA $(=6 / 6)$ the proportion of "pass" for the reading accuracy test was much lower (52.9\%) than in the children with lower dVA (81.8\%). Furthermore, the proportion of "requires follow up" and "fail" were higher than in the children with lower dVA (Figure 1B). This was also the case for reading speed test results (see Figure $1 \mathrm{C}$ for details). 
A

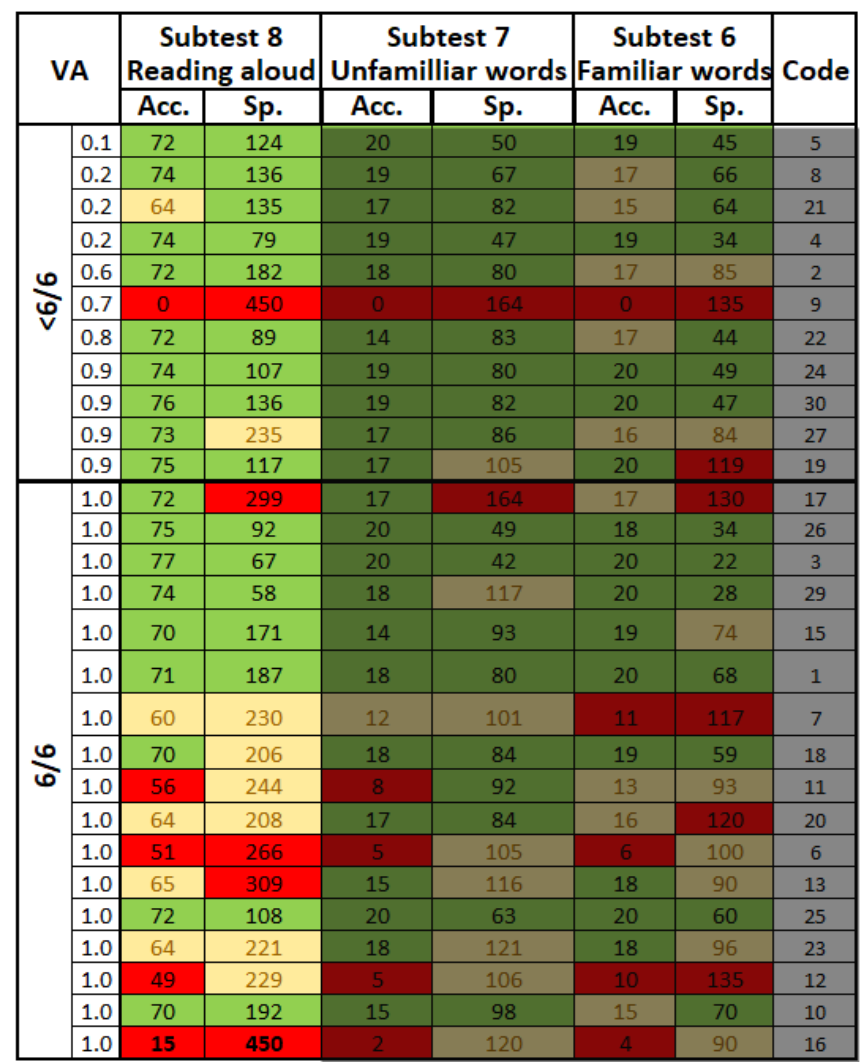

B
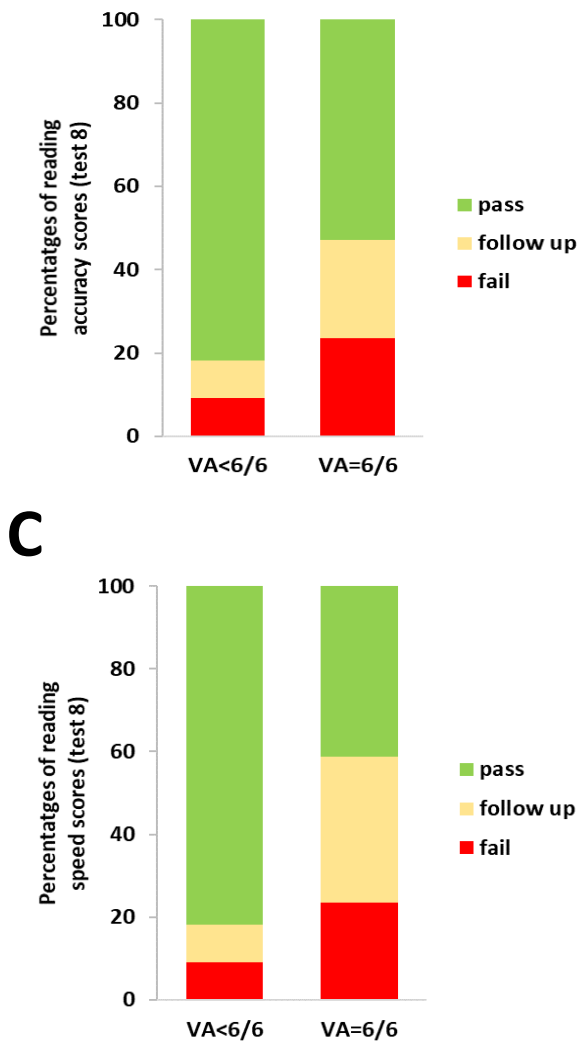

Figure 1. Reading performance according to VA performance. (A) Raw reading scores data sorted according to dVA (left column) with lower dVA scores $(<6 / 6, n=11)$ above the bold black line and normal dVA $(=6 / 6, n=17)$ below it. Each row represents dVA and reading tests results of one child. Red represents a "fail" grade, yellow represents "requires follow up" and green a "pass" grade according to the national standards (see Results for details). (B) Proportions of reading accuracy categorization scores of subtest 8 for poorer dVA ( $<6 / 6$ on left, $n=11$ ) and for normal dVA ( $=6 / 6$ on right, $n=17)$. (C) Same analysis as in (B) but for reading speed. Note that dVA was not predictive of reading performance as children with poorer dVA did not perform less accurately or slower on reading tests than children with normal dVA (see Results).

\section{Oculomotor screening}

King-Devick (KD) and the developmental eye movement test (DEM) are two well-established brief optometric screening tools whose saccadic demands on the visual system predominantly mimic those that reading entails. We therefore expected that in contrast to dVA, performance on King-Devick or the DEM would be predictive of reading performance, in either accuracy, speed, or both. 


\section{King-Devick}

We first correlated the reading speed and accuracy performance (subtest no. $8^{21}$ ) with King-

Devick performance. We found, as we hypothesized, King-Devick accuracy was indeed predictive of reading accuracy $(R=-0.51, t(26)=-3.02, p=0.0056)$ as was King-Devick speed of reading speed $(\mathrm{R}=0.62, \mathrm{t}(26)=3.98, \mathrm{p}=0.00050)$. Figure $2 \mathrm{~A}$ presents reading performance across 3 reading subtests (as described above, see distance VA section and Figure 1) sorted by King-Devick speed. As can be seen, faster King-Devick scores were associated with superior reading speed. Here too we compared the proportions of "pass", "requires follow up" and "fail" scores for reading speed according to national reading standards (subtest 8) between children with higher KD speed and within children with lower KD speed. We found that amongst children with slower King-Devick performance the proportion of "fail" for the reading speed test was four times higher $(28.6 \%)$ than in the children with faster King-Devick performance (7.1\%). Furthermore, the proportion of "pass" was higher in the children with faster King-Devick performance (71.4\%) than amongst those with slower King-Devick performance (42.9\%) (Figure 2B). Figure $2 \mathrm{C}$ presents reading performance across the 3 reading subtests $(6,7$ and 8$)$, sorted by King-Devick accuracy. Here too higher King-Devick accuracy scores were associated with higher reading accuracy. As can be seen in Figure 2D, the proportion of reading accuracy classification scores ("pass", "requires follow up" and "fail") amongst King-Devick upper performance was superior to that in the lower King-Devick accuracy performance (more "pass", less "fail", see Figures 2D for details). 
A

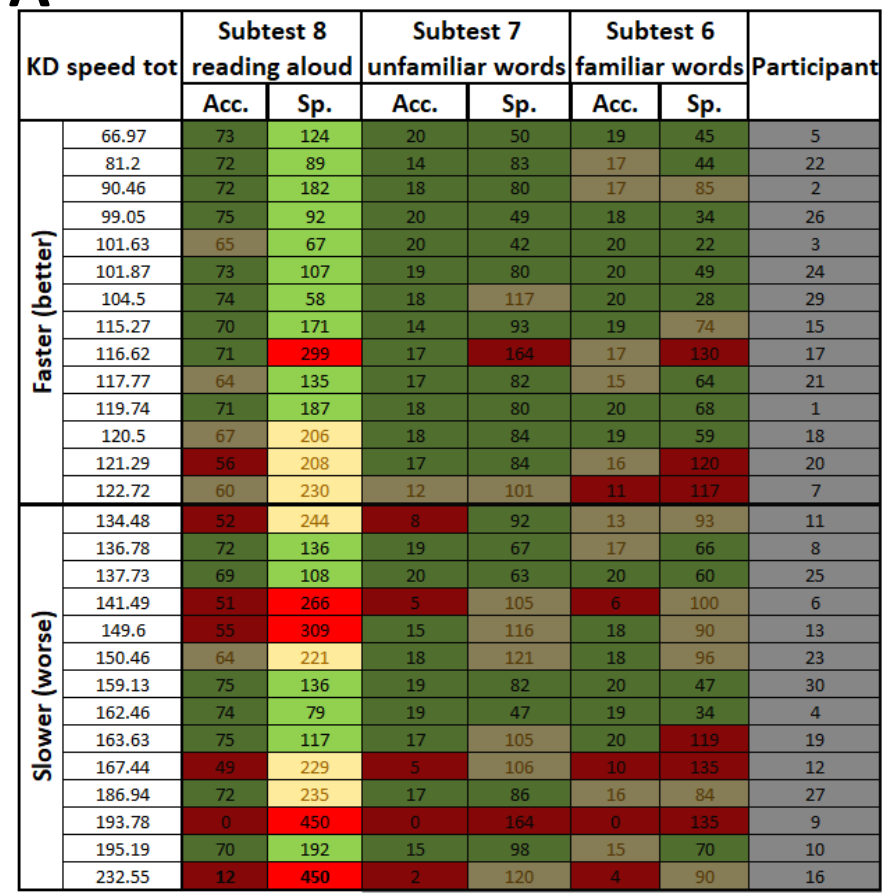

C

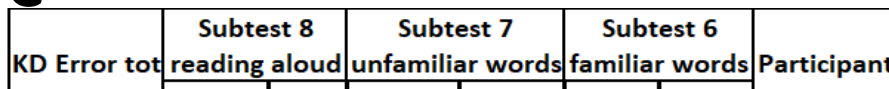

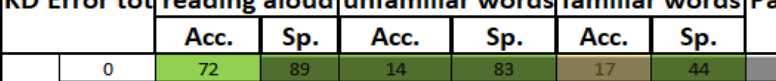

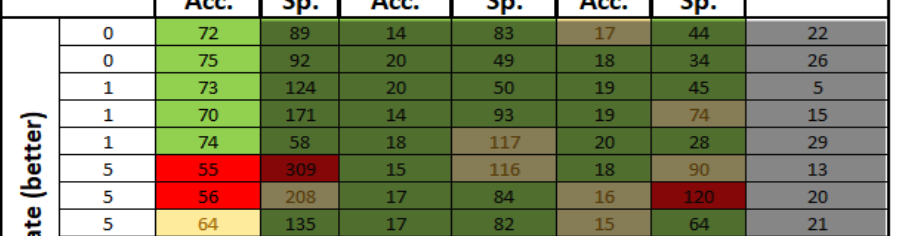

离

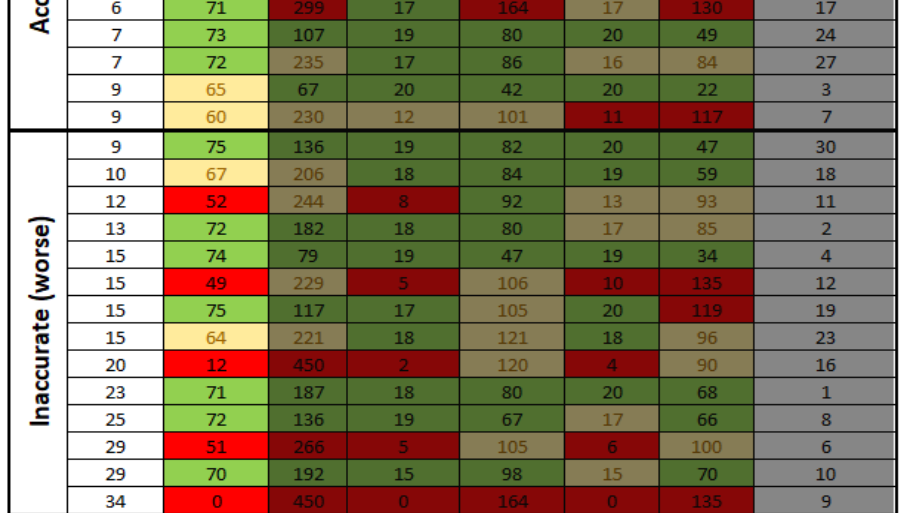

B

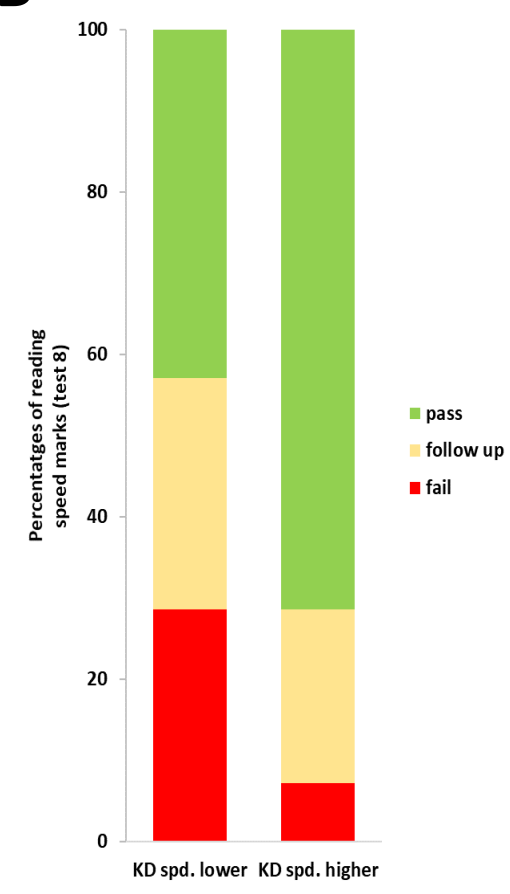

D

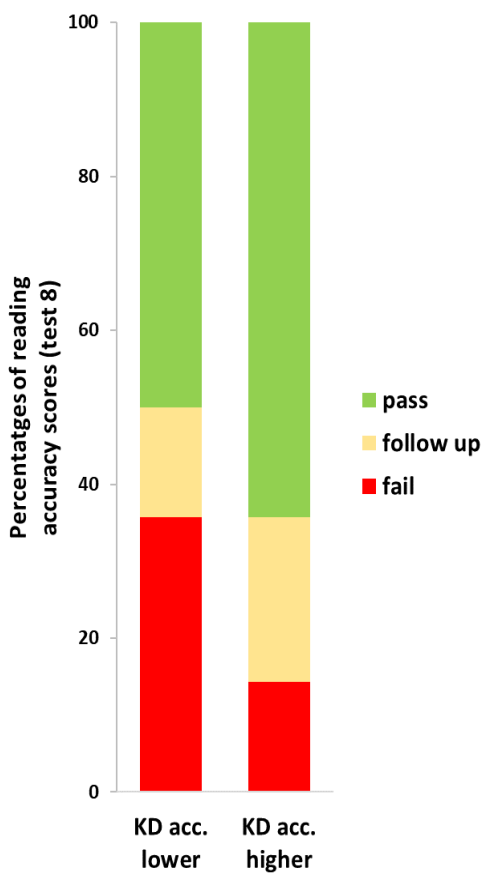

Figure 2. Reading performance according to King-Devick performance. (A) The same raw reading data scores as in Figure 1 but now sorted by King-Devick speed (on left column) with faster scores ( $n=14)$ above the bold black line and slower scores $(n=14)$ below it. Same conventions as in Figure 1. (B) Similar to Figure 1, proportions of reading accuracy categorization scores (same colors as in A) of subtest 8 for children with less accurate King-Devick performance (on left) and for children with more accurate King- 
Devick performance (on right). (C) As in panel A but reading data sorted according to King-Devick accuracy (left column); more accurate KD scores above the bold line, less accurate scores below it. (D) Same analysis and presentation as in (B) but for reading speed. Note that King-Devick accuracy and speed scores were each predictive of reading speed and accuracy performance (see Results for details).

\section{DEM}

As with the KD test, we wanted to evaluate if the DEM performance would act as an effective predictor for reading performance ability. To achieve this we correlated the reading speed and accuracy performance (subtest $8^{21}$ ) with DEM performance. Similar to King-Devick, as we hypothesized, DEM accuracy and speed performance were predictive, respectively, of reading accuracy $(R=-0.43, t(26)=-2.46, p=0.021)$ and speed $(R=-0.48, t(26)=2.80, p=0.0094)$. Figure $3 A$ presents reading performance across the 3 reading subtests $(6,7$, and 8 , see above) sorted according DEM speed. As can be seen, superior reading speed scores were associated with faster DEM scores. Similar to King-Devick analyses and as can be seen in Figure 3B, the proportion of "fail" for the reading speed test amongst children with slower DEM performance was four times higher (28.6\%) than in the children with faster DEM performance (7.1\%). Furthermore, the proportion of "pass" for the reading speed in the children with faster DEM performance $(78 \%)$ was twice as high as the proportion amongst those with slower DEM performance (35.7\%). Figure 3C presents reading performance across the 3 reading subtests $(6$, 7 and 8), sorted by DEM accuracy. Here too superior reading accuracy co-occurs with higher DEM accuracy scores. The proportions of reading marks categories ("pass", "requires follow up", or "fail") based on national reading standards ${ }^{21}$ (subtest 8) are presented in Figure 3D with low DEM accuracy (left, $n=14$ ) and higher DEM accuracy group (right, $n=14$ ). These show that better reading (more "pass" marks and less "fail" marks) is evident in the higher DEM accuracy group (right). Similar to previous studies by Rouse ${ }^{30}$ and others, we did not find significant correlation between DEM ratio and reading speed $(R=0.10, t(26)=0.51, p=0.61)$ or reading accuracy $(R=-$ $0.12, \mathrm{t}(26)=-0.62, \mathrm{p}=0.54)$. 
A

\begin{tabular}{|c|c|c|c|c|c|c|c|c|}
\hline \multicolumn{2}{|c|}{ DEM Sp. } & \multicolumn{2}{|c|}{$\begin{array}{c}\text { Subtest } 8 \\
\text { reading aloud }\end{array}$} & \multicolumn{2}{|c|}{$\begin{array}{c}\text { Subtest } 7 \\
\text { unfamiliar words }\end{array}$} & \multicolumn{2}{|c|}{\begin{tabular}{|c|} 
Subtest 6 \\
familiar words \\
\end{tabular}} & \multirow[t]{2}{*}{ Participant } \\
\hline & & Acc. & Sp. & Acc. & Sp & Acc. & Sp. & \\
\hline \multirow{14}{*}{ 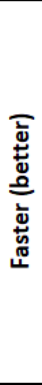 } & 43.57 & 72 & 182 & 18 & 80 & 17 & 85 & 2 \\
\hline & 70.61 & 72 & 89 & 14 & 83 & 17 & 44 & 22 \\
\hline & 75.69 & 75 & 92 & 20 & 49 & 18 & 34 & 26 \\
\hline & 78.87 & 73 & 124 & 20 & 50 & 19 & 45 & 5 \\
\hline & 90.66 & 74 & 58 & 18 & 117 & 20 & 28 & 29 \\
\hline & 94.74 & 65 & 67 & 20 & 42 & 20 & 22 & 3 \\
\hline & 94.75 & 60 & 230 & 12 & 101 & 11 & 117 & 7 \\
\hline & 100.24 & 56 & 208 & 17 & 84 & 16 & 120 & 20 \\
\hline & 100.53 & 70 & 171 & 14 & 93 & 19 & 74 & 15 \\
\hline & 102.21 & 72 & 136 & 19 & 67 & 17 & 66 & 8 \\
\hline & 103.02 & 64 & 135 & 17 & 82 & 15 & 64 & 21 \\
\hline & 104.20 & 71 & 187 & 18 & 80 & 20 & 68 & 1 \\
\hline & 112.03 & 73 & 107 & 19 & 80 & 20 & 49 & 24 \\
\hline & 119.38 & 71 & 299 & 17 & 164 & 17 & 130 & 17 \\
\hline \multirow{14}{*}{ 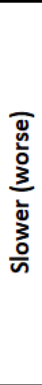 } & 119.53 & 64 & 221 & 18 & 121 & 18 & 96 & 23 \\
\hline & 123.01 & 67 & 206 & 18 & 84 & 19 & 59 & 18 \\
\hline & 131.06 & 75 & 136 & 19 & 82 & 20 & 47 & 30 \\
\hline & 143.53 & 52 & 244 & 8 & 92 & 13 & 93 & 11 \\
\hline & 144.33 & 55 & 309 & 15 & 116 & 18 & 90 & 13 \\
\hline & 152.52 & 51 & 266 & 5 & 105 & 6 & 100 & 6 \\
\hline & 178.97 & 69 & 108 & 20 & 63 & 20 & 60 & 25 \\
\hline & 196.18 & 72 & 235 & 17 & 86 & 16 & 84 & 27 \\
\hline & 201.30 & 74 & 79 & 19 & 47 & 19 & 34 & 4 \\
\hline & 238.21 & 49 & 229 & 5 & 106 & 10 & 135 & 12 \\
\hline & 243.14 & 0 & 450 & 0 & 164 & 0 & 135 & 9 \\
\hline & 251.20 & 75 & 117 & 17 & 105 & 20 & 119 & 19 \\
\hline & 300.00 & 70 & 192 & 15 & 98 & 15 & 70 & 10 \\
\hline & 300.00 & 12 & 450 & 2 & 120 & 4 & 90 & 16 \\
\hline
\end{tabular}

C

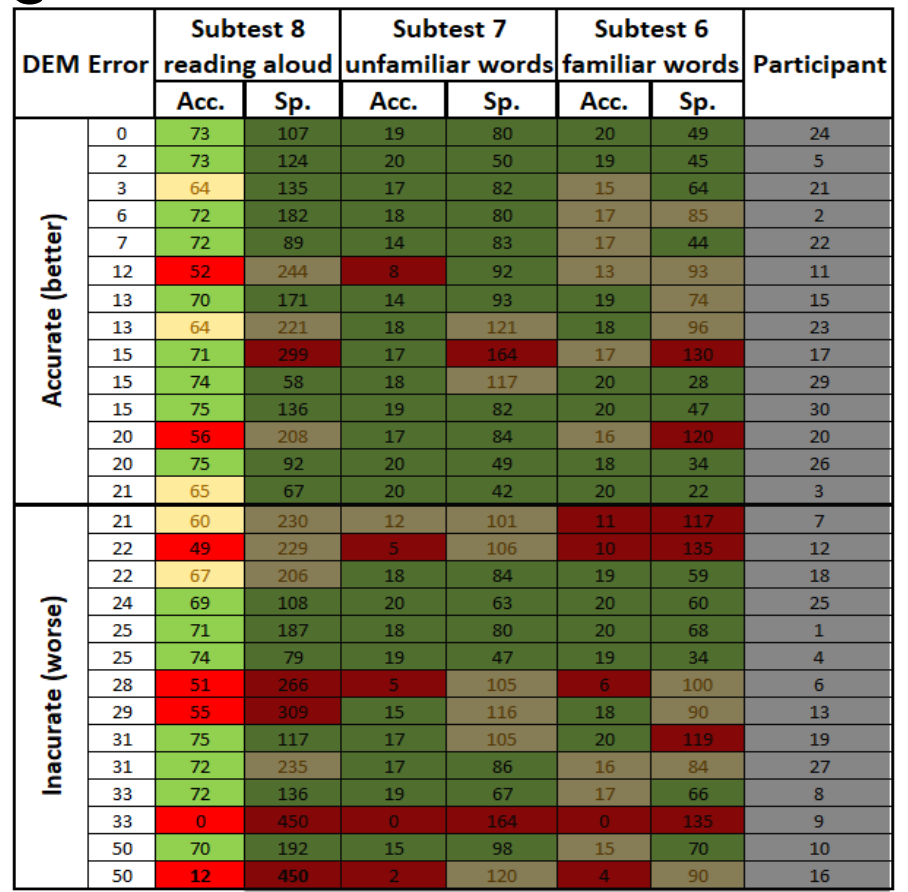

B

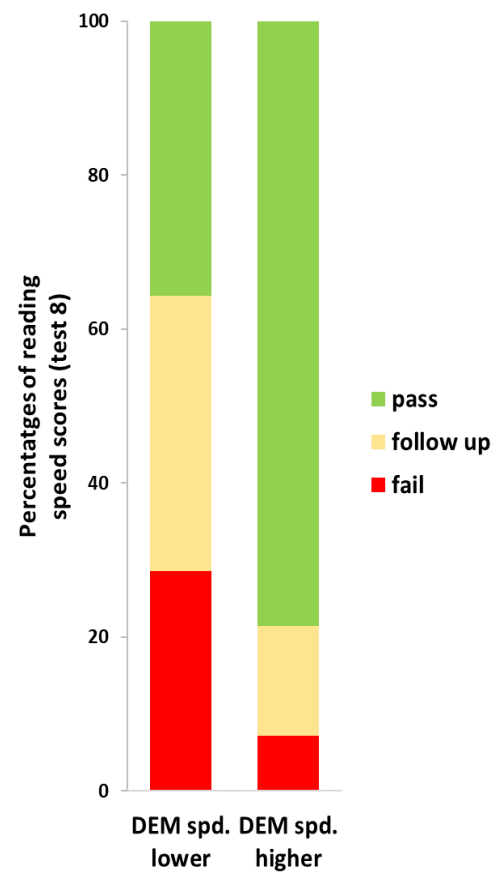

D

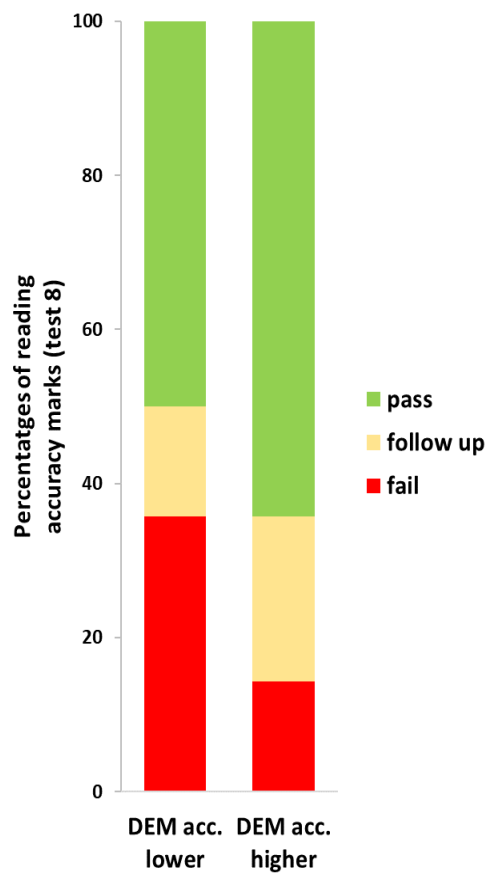

Figure 3. Reading performance according to DEM performance. (A) The same raw reading scores as in Figures 1-2 but here sorted according to DEM speed (on left column) with faster scores above the bold line $(n=14)$ and slower scores below it $(n=14)$. Conventions as in Figures 1 and 2. (B) Proportions of reading accuracy categorization scores of subtest 8 for the group of children with slower DEM performance (left) and for the group with faster DEM performance (right). (C) The raw reading scores sorted according to DEM accuracy (on left column) with more accurate performance $(n=14)$ above the bold line and less accurate performance $(n=14)$ below it. (D) Same analysis as in (B) but for reading 
accuracy. Note that DEM accuracy and speed scores were predictive of reading accuracy and speed (respectively, see Results).

\section{Comparing King-Devick and DEM}

Unlike the KD, the DEM also takes into account the child's rapid automatic naming ability (RAN).

Furthermore, only the DEM adjusts the time measured according to the level of accuracy. Both tests however, despite their differences, are standardized optometric tests designed to assess horizontal eye movements. As they were both predictive of reading scores, with neither being superior (correlation difference between KD accuracy with reading accuracy vs. DEM accuracy with reading accuracy was not significantly different: $Z=0.36, p=0.7188$, two tailed; correlation difference between KD speed with reading speed vs. DEM speed with reading speed was not significantly different: $Z=-0.66, p=0.5093$, two tailed), we examined whether these tests single out the same children or perhaps different ones with respect to reading ability. Since in our analysis we focused on comparing the reading scores in the upper and lower optometric performance halves of the class (for different optometric tests), we examined the interchangeability of individual children between these two tests with respect to upper and lower performance halves of the class. We split the class into two halves based on better/poorer accuracy or speed for each of the two tests. We then evaluated the number of children that were not classified in the same half of the class by the two tests (assigned to the upper half by one test and the lower half by the other test or vice versa). Over $10 \%$ of the children in our study that were assigned to the lower half of the class according to DEM accuracy were assigned to the upper half according to KD accuracy. For speed, the mismatch between the two oculomotor tests was of one child (in our cohort $>3.5 \%$ ). Overall, administering both KD and DEM tests may be beneficial to avoid missing children with OMD that could be at risk of struggling with reading acquisition. 


\section{NSUCO}

NSUCO is a well-established optometric test aimed at detecting gross oculomotor deficits. We were interested in assessing whether the subsection of NSUCO saccadic head movement scores can also be predictive of reading performance as we hypothesized they are likely to cause reading inaccuracy (see Methods). The correlation between the NSUCO saccadic head movement subtest and reading accuracy approached significance $(R=-0.32, t(26)=1.74$, $p=0.094)$. Figure $4 A$ presents reading performance across the 3 reading subtests $(6,7$ and 8$)$ sorted according NSUCO subtest head movement, and Figure 4B the proportions of the reading categorization marks based on national reading standards ${ }^{21}$ (subtest 8 ) for the superior NSUCO performers $(n=18)$ and for lower NSUCO performers $(n=10)$. We found that amongst children with little or no head movement the proportion of "fail" was zero (see Figure 4B right column). In contrast, amongst children who struggled to dissociate oculomotor and head movement, 38.9\% failed the reading accuracy test (see Figure 4B left column). Furthermore, the proportion of "pass" was much higher with better NSUCO performance (80\%) than amongst those with poorer NSUCO performance (44.4\%) (Figure 4B). 
A

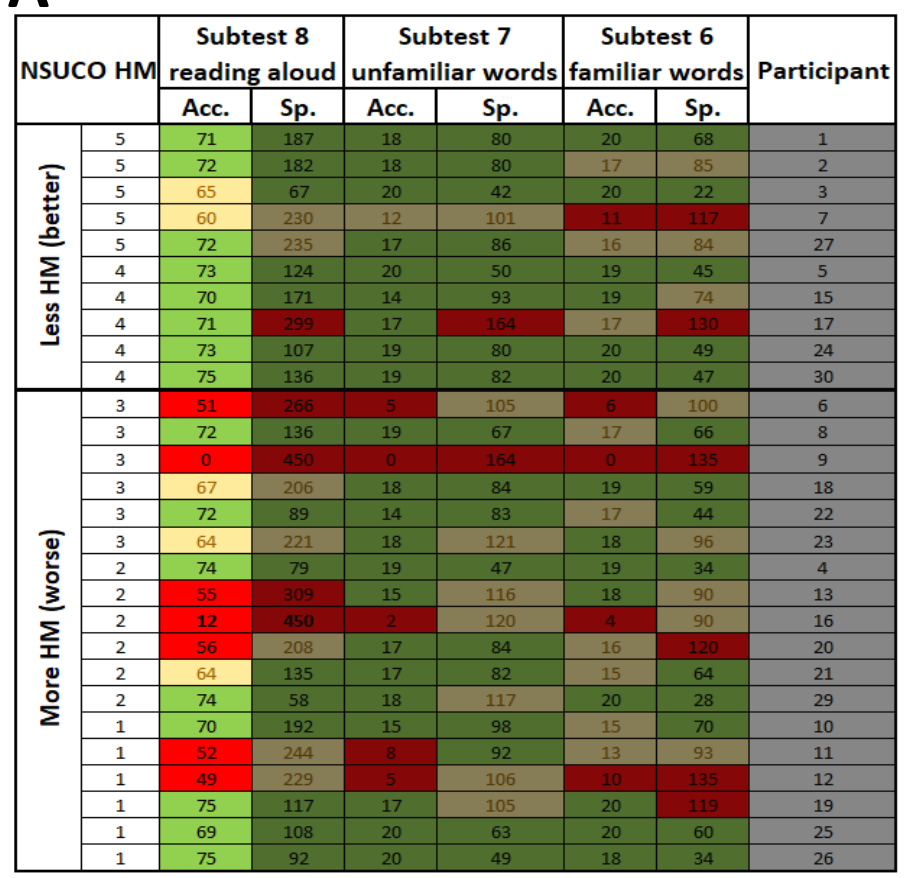

B

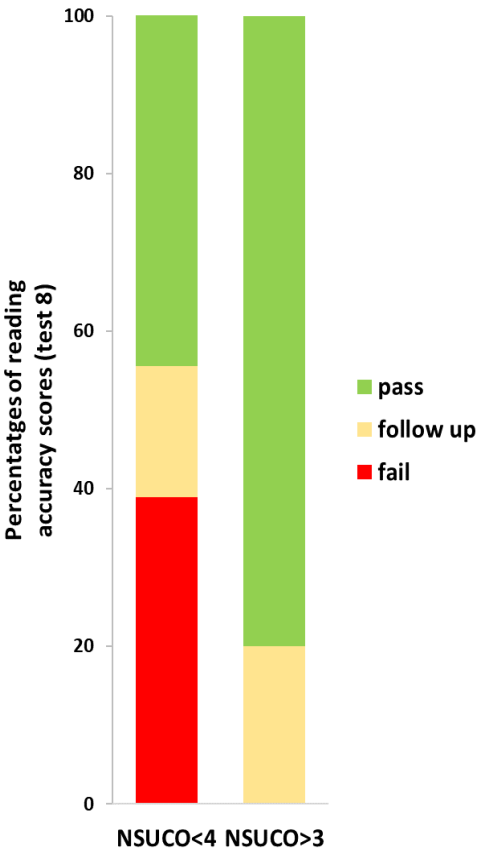

Figure 4. Reading performance according to NSUCO head movement performance. (A) The raw reading scores (as in previous figures) but sorted according to NSUCO head movement scores (left column) with better (less head movement) scores (of $4-5)$ above the bold line $(n=10)$ and worse (more acute head movement scores of 1-3) below it ( $n=18)$. Conventions as in Figures 1-3. (B) Proportions of reading accuracy categorization scores of subtest 8 for children who displayed more head movement (worse oculomotor control) on the left and less head movement (better oculomotor control) on the right. The correlation between the ability to dissociate head moment and ocular movement during the NSUCO test was marginally predictive of reading accuracy (see Results).

\section{Discussion}

In this study we investigated how different visual abilities are related to reading performance in a group of first grade children. We found that despite its prevalent use, dVA was not predictive of reading performance. More importantly, we found that in line with our hypothesis saccadic related King-Devick and DEM optometric tests were significantly predictive of reading performance (both accuracy and speed). The NSUCO saccadic head movement subtest also provided good indication of reading accuracy.

In the literature, the relationship between dVA and reading is still unclear. We found, in line with our hypothesis, that dVA is not critical for reading acquisition. This may be because reading 
acquisition is most often performed at close viewing distances and uses large font sizes. Bruce et al. report finding a correlation between dVA and developing literacy skills at the age of 4-5. In their study they used the Woodcock Reading Mastery Tests-Revised (WRMTS-R) ${ }^{31}$, perhaps due to the young children's pre-reading age. The correlation that they report is based on a subtest of the WRMTS-R that demands letter recognition and not whole word or sentence reading. Such visually local letter recognition is likely to rely on similar functions as VA testing, and therefore it may not be surprising that such measures correlate. However, this does not necessarily predict reading ability that relies on additional non-local multimodal processes. It is interesting that in a review by De Rose the WRMTS-R test was criticized as a tool for assessing reading ability ${ }^{32}$. A recent review by Hopkins et al. on dVA and reading ability concludes that the impact of dVA on reading ability is still unclear. They conclude that while there may be some evidence supporting a connection between dVA and reading ability, it is inconclusive and further research is necessary $^{33}$. In contrast to the above studies and in line with our hypothesis and results, several other studies report that dVA is not associated with reading ability. One longitudinal study assessing 1143 nine and ten year olds found that there was no correlation between dVA and academic school performance ${ }^{34}$. However, that study did not directly assess the relationship between dVA and reading. In fact in their analysis they regressed reading related measures out. Additionally, as the age of the children in their study was 9 and 10, it is difficult to draw any inferences from these findings about reading acquisition that typically occurs at earlier age. Vinuela-Navarro et al. found that dVA was not predictive of reading skills ${ }^{35}$. While we are aware that dVA is important amongst first graders for multiple purposes (seeing the board, reading the teacher's lips, social communication), our results support the view that dVA alone is not a sufficiently effective screening tool of visual skills necessary for reading acquisition. 
We hypothesized that oculomotor control would be predictive of reading speed and accuracy and this was significantly supported by our findings with both DEM and KD, while the NSCUCO results were only marginally significant. The idea that oculomotor control is related to reading ability is also in line with a previous study by Hoffman suggesting an estimated increase of $400 \%$ in the prevalence of OMD between poor versus good readers ${ }^{3}$. Serdjukova et al., while not assessing accuracy, also found the DEM speed to be predictive of reading speed ability ${ }^{36}$. A different study assessing the relation between reading ability and saccades reports that these are not correlated ${ }^{35}$. However, in that study, eye movements were assessed while viewing animated stimuli and cartoon characters which are very different from text (e.g. in spatial and spectral structure) and are likely to rely on different brain and oculomotor patterns than those that reading relies on. While there are differences between the $D E M$ and KD, both are standardized optometric tests assessing horizontal eye movements. As of yet, it is unclear if any one of them is a more effective screening tool for saccadic control necessary for reading than the other ${ }^{17}$. We found that both tests were significantly predictive of reading ability but did not single out precisely the same group of children that failed. Maples and Ficklin report that the KD test is predictive of the NSUCO head movement subtest ${ }^{37}$. Their study did not correlate these results with reading. In another study however, similar to our findings, Maples and Ficklin do report that higher NSUCO scores are predictive of improved reading ability ${ }^{38}$. The fact that Maples and Ficklin found the relation between NSUCO and reading ability to be significant while our results were only marginally significant may be due to differences in cohort sizes between the studies. The NSUCO head movements subtest checks for head movements during ocular saccades which are not assessed by the DEM or King-Devick. Furthermore, unlike the DEM or $K D$, the NSUCO is a nonverbal test and assesses oculomotor function in a different manner than the DEM or KD. We therefore suggest that all three portable, cheap and time efficient OMD 
tests be administered in order to detect any possible aberrant findings (accuracy, time to complete) that may cause reading difficulty.

Hebrew for early readers is a highly transparent (aka shallow) language. This means that most of the time it is read as it is written due to a consistent relationship between its graphemes and phonemes ${ }^{39-41}$. Languages with deep orthography require the reader to take a more global, whole word or semantic approach to reading. As the Hebrew reader becomes more fluent, the diacritics (pronunciation symbols) are dropped and the reading takes on a more semantic processing form. The current study's reading assessment was performed using Hebrew with diacritics. While our results were obtained using a shallow/transparent orthography, we hypothesize that OMD also affects deep orthographic languages. This is supported by a recent study by Kelly et al. using a deep orthographic language (English) that suggests that slow reading speed is related to poor saccadic control and to fixation instability ${ }^{42}$. A review by Kulp and Schmidt also suggests a correlation between oculomotor dysfunction and reading disability in deep orthographic languages such as English ${ }^{43}$. Our oculomotor assessments (both King-Devick and DEM) were administered, as instructed, from left to right so that we could compare our results to these tests' normative data, and also since these tests are based on numeric elements that are typically read from left to right. However, since Hebrew is read in the opposite direction (right to left), all the reading assessments were performed in the opposite direction in a right to left manner. Lastly, while Garzia et al. found language did not affect speed of DEM performance $^{26}$, Fernandez-Velazquez \& Fernandez-Fidalgo found that in 6 year old Spanish speakers DEM was significantly slower relative to the English-based norms ${ }^{44}$. We cannot rule out a possibility that a change in directionality or language may have influenced our results thus strengthening or weakening our findings. However, such directionality or language advantage/disadvantage, if present, was equal across all the children in our study. While we 
hypothesize that our results may be generalized to additional reading languages with both deep and shallow orthographies and right to left reading, since our study was based on findings from 28 children, further research is necessary to assess how OMD affects additional cohorts.

There are several theories in the literature as to the cause of poor or regressive saccades during reading and these are reviewed by Vitu and McConkie and Rayner ${ }^{45,46}$. One of these theories claims corrective saccades are to remediate an inaccurate previous forward saccade which caused the eyes to land incorrectly. A second theory suggests that eyes often make regressive saccades as a result of leaving the word before it was identified or due to incorrect ocular positioning on the fixated word which complicates the identification of that word. A third theory proposes that the regressive saccades are necessary to enable the reader to reread a section of text when faced with comprehension challenges. Our data that shows significant correlation between OMD-related measures and reading performance do not necessarily refute or support any of these theories that may each may come into play under different circumstances. It is worth noting that not all poor readers in our study displayed OMD-related findings. This supports our hypothesis that OMD may often be a possible cause of reading difficulties and not a result of reading difficulty. People with oculomotor deficits have been shown to benefit from oculomotor related treatments that typically reduce OMD related difficulties ${ }^{15,47-51}$. Therefore, screening for $\mathrm{OMD}$ in early readers and possibly consulting with specialists to consider dedicated support could be an effective policy for improving scholastic performance across school aged children.

\section{Conclusion}

OMD's prevalence in school aged good readers is estimated to be roughly one in five children while almost all children diagnosed with a reading disability are estimated to suffer from $\mathrm{OMD}^{3}$. 
While future assessments of the relation between OMD related measures and reading performance are necessary, our study suggests that reading among Hebrew speaking children is significantly associated with OMD-related measures but not with dVA. The British Dyslexia Association has already included full visual motor and visual perceptual evaluations as a prerequisite to dyslexia testing ${ }^{52}$. We are hoping that our study will support enabling further paradigm shifts in additional countries for both evaluating vision skills of early readers as well as for potential treatment protocols of those pupils struggling to acquire efficient reading skills. This is important since the primary scholastic goal of first grade is the achievement of accurate reading which plays a key role and affords the acquisition of many additional skills later in life $\mathrm{e}^{53}$.

\section{Acknowledgements}

We thank RR for her assistance with the reading assessments. SGD was supported by ISF individual grant no. $1485 / 18$ to SGD.

\section{Author contributions}

AP and SGD designed the retrospective analysis of the data after AP performed the optometric examinations (while being blind to the children's scholastic and reading assessments). AP retrospectively analyzed the data. AP and SGD interpreted the results. AP and SGD wrote the paper.

\section{Competing interests statement}

All authors (AP and SGD) declare that they do not have any conflicts of interests.

\section{Data availability}

No data sets were generated or analyzed during the current study. 


\section{Bibliography}

1. The Annie E. Casey Foundation. Kids Count Data Book State Trends in Child Well-Being. Baltimore MD; 2019.

2. Hernandez DJ. Double Jeopardy: How Third-Grade Reading Skills and Poverty Influence High School Graduation. Annie E Casey Found. 2011.

3. Hoffman LG. Incidence of vision difficulties in children with learning disabilities. J Am Optom Assoc. 1980;51(5):447-451.

4. Lieberman S. The prevalence of visual disorders in a school for emotionally disturbed children. J Am Optom Assoc. 1985;56(10):800-803.

5. Quaid P, Simpson T. Association between reading speed, cycloplegic refractive error, and oculomotor function in reading disabled children versus controls. Graefe's Arch Clin Exp Ophthalmol. 2013;251(1):169-187.

6. Teramoto W, Kawano S, Mori S, Sekiyama K. Word scanning in native and non-native languages: insights into reading with declined accommodation. Exp Brain Res. 2019;237(9):2411-2421.

7. Dusek W, Pierscionek BK, Mcclelland JF. A survey of visual function in an Austrian population of school-age children with reading and writing difficulties. BMC Ophthalmol. 2010;10:16.

8. Borsting E, Mitchell GL, Kulp MT, et al. Improvement in Academic Behaviors After Successful Treatment of Convergence Insufficiency. Optom Vis Sci. 2012;89(1):12-18.

9. Cooper J, Selenow A, Ciuffreda KJ, et al. Reduction of asthenopia in patients with convergence insufficiency after fusional vergence training. Am J Optom Physiol Opt. 1983;60(12):982-989.

10. Cooper J, Feldman J, Selenow A, et al. Reduction of asthenopia after accommodative facility training. Am J Optom Physiol Opt. 1987;64(6):430-436.

11. Krieber M, Bartl-Pokorny KD, Pokorny FB, et al. The Relation between Reading Skills and Eye Movement Patterns in Adolescent Readers: Evidence from a Regular Orthography. PLoS One. 2016;11(1):e0145934. doi:10.1371/journal.pone.0145934

12. Eden GF, Stein JF, Wood HM, Wood FB. Differences in eye movements and reading problems in dyslexic and normal children. Vision Res. 1994;34(10):1345-1358.

13. Biscaldi M, Gezeck S, Stuhr V. Poor saccadic control correlates with dyslexia. Neuropsychologia. 1998;36(11):1189-1202.

14. Blythe HI, Joseph HSSL. Children's eye movements during reading. Oxford Handb. August 2011.

15. Dodick D, Starling AJ, Wethe J, et al. The Effect of In-School Saccadic Training on Reading Fluency and Comprehension in First and Second Grade Students. J Child Neurol. 2017;32(1):104-111. 
16. Sloot $\mathrm{F}$, Hoeve $\mathrm{HL}$, de Kroon $\mathrm{L}$, et al. Inventory of current $\mathrm{EU}$ paediatric vision and hearing screening programmes. J Med Screen. 2015;22(2):55-64.

17. Heick JD, Bay C, McLeod V, Tamara C. Evaluation of vertical and horizontal saccades using the developmental eye movment test compared to the King-Devick test. Int J Sport Phys Ther. 2018;13(5):808-818.

18. Tassinari JT, DeLand P. Developmental Eye Movement Test: reliability and symptomatology. Optom - J Am Optom Assoc. 2005;76(7):387-399.

19. Facchin A, Maffioletti S. The Reliability of the DEM Test in the Clinical Environment. Front Psychol. 2018;9:1279.

20. Maples WC, Atchley J, Ficklin T. Northeastern State University College of Optometry's oculomotor norms. J Behav Optom. 1992;3:143-150.

21. Mast I, Bar-On A and YS. Grade 1 Reading and Writing Exam Teacher's Guide. The National Authority for Assessment and Evaluation in Education; 2015.

22. McLeod H, Chaglasian E. Effect of Room Illumination on Manifest Refraction. Optom Educ. 2018;44(2).

23. Bharadwaj SR, Malavita M, Jayaraj J. A psychophysical technique for estimating the accuracy and precision of retinoscopy. Clin Exp Optom. 2014;97(2):164-170.

24. Scheiman M, Mitchell GL, Cotter S, et al. A Randomized Clinical Trial of Treatments for Convergence Insufficiency in Children. Arch Ophthalmol. 2005;123(1):14.

25. Norton ES, Wolf M. Rapid Automatized Naming (RAN) and Reading Fluency: Implications for Understanding and Treatment of Reading Disabilities. Annu Rev Psychol. 2012;63(1):427-452.

26. Garzia RP, Richman JE, Nicholson SB, Gaines CS. A new visual-verbal saccade test: the development eye movement test (DEM). J Am Optom Assoc. 1990;61(2):124-135.

27. Lieberman S, Cohen AH, Rubin J. NYSOA K-D test. J Am Optom Assoc. 1983;54(7):631-637.

28. Maples W. NSUCO Oculomotor Test Manual | OEP Foundation. Optom Ext Progr Found. 1995.

29. Groto I. Advance Guidelines (No: 12/2015)for Public Health Services in the Educational System September 2014 (Ref: 58516915). Jerusalem: Ministry of Health; 2014:68501914.

30. Rouse MW, Nestor EM, Parot CJ, DeLand PN. A reevaluation of the Developmental Eye Movement (DEM) test's repeatability. Optom Vis Sci. 2004;81(12):934-938.

31. Bruce A, Fairley L, Chambers B, Wright J, Sheldon TA. Impact of visual acuity on developing literacy at age 4-5 years: A cohort-nested cross-sectional study. BMJ Open. 2016;6(2).

32. De Rose M. A Review of the Woodcock Reading Mastery Test-Revised (WRMT-R). TESL CANADA J. 1999;16(2):86-93.

33. Hopkins S, Narayanasamy S, Vincent SJ, Sampson GP, Wood JM. Do reduced visual acuity and refractive error affect classroom performance? Clin Exp Optom. 2019:1-12. 
34. Dirani M, Zhang X, Goh LK, Young TL, Lee $P$, Saw SM. The role of vision in academic school performance. Ophthalmic Epidemiol. 2010;17(1):18-24.

35. Vinuela-Navarro V, Erichsen JT, Williams C, Woodhouse JM. Saccades and fixations in children with delayed reading skills. Ophthalmic Physiol Opt. 2017;37(4):531-541.

36. Serdjukova J, Ekimane L, Valeinis J, Skilters J, Krumina G. How strong and weak readers perform on the Developmental Eye Movement test (DEM): norms for Latvian schoolaged children. Read Writ. 2017;30(2):233-252.

37. Maples W, Ficklin T. Test-retest reliability of the King-Devick Saccade and the NSUCO ocular motor tests. J Behav Optom. 1991;3:209-214.

38. Maples W, T F. A preliminary study of the oculomotor skills of learning-disabled, gifted and normal children. Dev, Optom Vis Dev. 1989;20:9-14.

39. Bar-Kochva I, Breznitz Z. Does the reading of different orthographies produce distinct brain activity patterns? An ERP study. PLoS One. 2012;7(5):1-8.

40. Frost R. Prelexical and postlexical strategies in reading: Evidence from a deep and a shallow orthography. J Exp Psychol Learn Mem Cogn. 1994;20(1):116-129.

41. Schiff R. Shallow and Deep Orthographies in Hebrew: The Role of Vowelization in Reading Development for Unvowelized Scripts. J Psycholinguist Res. 2012;41:409-424.

42. Kelly KR, Jost RM, De La Cruz A, et al. Slow reading in children with anisometropic amblyopia is associated with fixation instability and increased saccades. In: Journal of AAPOS. Vol 21. Mosby Inc.; 2017:447-451.e1.

43. KULP MT, SCHMID PP, Schmidt PP. Effect of Oculomotor and Other Visual Skills on Reading Performance: A Literature Review. Vol 73. Lippincott Williams and Wilkins; 1996:283-292.

44. Fernandez-Velazquez F, Fernandez-Fidalgo M. Do DEM test scores change with respect to the language? norms for spanish-speaking population. Optom Vis Sci. 1995;72(12):902906.

45. Vitu F, McConkie GW. Regressive Saccades and Word Perception in Adult Reading. In: Reading as a Perceptual Process. North-Holland: Elsevier; 2000:301-326.

46. Rayner K. Eye Movements in Reading and Information Processing: 20 Years of Research. Psychol Bull. 1998;124(3):372-422.

47. Young B, Pollard T, Paynter S, Cox R. Effect of eye exercises in improving control of eye movements during reading. J Optom Vis Dev. 1982;13:4-7.

48. Rounds B, Manley C, Norris R. The effect of oculomotor training on reading efficiency. Coll Optom. 1988.

49. Thiagarajan P, Ciuffreda KJ, Capo-Aponte JE, Ludlam DP, Kapoor N. Oculomotor neurorehabilitation for reading in mild traumatic brain injury (mTBI): an integrative approach. NeuroRehabilitation. 2014;34(1):129-146.

50. Watabe T, Suzuki H, Abe M, Sasaki S, Nagashima J, Kawate N. Systematic review of visual 
rehabilitation interventions for oculomotor deficits in patients with brain injury. Brain inj. 2019:1-5.

51. Nazir M, Nabeel T. Effects of Training of Eye Fixation Skills on the Reading Fluency of Children with Oculomotor Dysfunction. Pakistan J Educ. 2019;36(1):61-80.

52. British Dyslexia Association. How to apply for an assessment - British Dyslexia Association. https://www.bdadyslexia.org.uk/services/assessments/diagnosticassessments/application-information. Accessed September 22, 2019.

53. Hulme C, Snowling MJ. Reading disorders and dyslexia. Curr Opin Pediatr. 2016;28(6):731-735. 